\title{
TB Meningitis in HIV-Positive Patients in Europe and Argentina: Clinical Outcome and Factors Associated with Mortality
}

\author{
Anne Marie W. Efsen, ${ }^{1}$ Alexander M. Panteleev, ${ }^{2}$ Daniel Grint, ${ }^{3}$ Daria N. Podlekareva, \\ Anna Vassilenko, ${ }^{4}$ Aza Rakhmanova, ${ }^{5}$ Indra Zeltina, ${ }^{6}$ Marcelo H. Losso, ${ }^{7}$ Robert F. Miller, ${ }^{8}$ \\ Enrico Girardi, ${ }^{9}$ Joan Caylá, ${ }^{10}$ Frank A. Post, ${ }^{11}$ Jose M. Miro, ${ }^{12}$ Mathias Bruyand, ${ }^{13,14}$ \\ Hansjakob Furrer, ${ }^{15}$ Niels Obel, ${ }^{16}$ Jens D. Lundgren, ${ }^{1,16}$ Amanda Mocroft, ${ }^{3}$ Ole Kirk, ${ }^{1,16}$ \\ and HIV/TB Study Group ${ }^{1}$
}

${ }^{1}$ Copenhagen HIV Programme, Rigshospitalet, Faculty of Health and Medical Sciences, Copenhagen University Hospital and University of Copenhagen, 2200 Copenhagen N, Denmark

${ }^{2}$ TB Hospital No. 2, Russian Federation, 195267 Saint Petersburg, Russia

${ }^{3}$ University College London, Royal Free Campus, London NW3 2PF, UK

${ }^{4}$ Belorusian State Medical University, Minsk 220002, Belarus

${ }^{5}$ St. Petersburg AIDS Centre, 193167 Saint Petersburg, Russia

${ }^{6}$ Infectology Centre of Latvia, 1006 Riga, Latvia

${ }^{7}$ Hospital JM Ramos Mejia, Servicio de Inmunocomprometidos, CP 1221 Buenos Aires, Argentina

${ }^{8}$ Centre for Sexual Health \& HIV Research, Mortimer Market Centre, University College London, London WC1E 6JB, UK

${ }^{9}$ Istituto Nazionale Malattie Infettive L Spallanzani, 00149 Rome, Italy

${ }^{10}$ Servicio de Epidemiología, Agencia de Salud Pública de Barcelona, CIBER Epidemiología y Salud Pública (CIBERESP), 08036 Barcelona, Spain

${ }^{11}$ King's College London School of Medicine, London SE5 9RS, UK

${ }^{12}$ Hospital Clinic-IDIBAPS, University of Barcelona, 08036 Barcelona, Spain

${ }^{13}$ Centre Hospitalier Universitaire (CHU) de Bordeaux, COREVIH Aquitaine, 33000 Bordeaux, France

${ }^{14}$ INSERM, ISPED, Centre Inserm U897-Epidemiologie-Biostatistique, 33000 Bordeaux, France

${ }^{15}$ Department of Infectious Diseases, Bern University Hospital and University of Bern, CH-3010 Bern, Switzerland

${ }^{16}$ Department of Infectious Diseases, Copenhagen University Hospital/Rigshospitalet, 2100 Copenhagen, Denmark

Correspondence should be addressed to Anne Marie W. Efsen; amw@cphiv.dk

Received 17 May 2013; Revised 12 September 2013; Accepted 30 September 2013

Academic Editor: Antonio Pacheco

Copyright ( 2013 Anne Marie W. Efsen et al. This is an open access article distributed under the Creative Commons Attribution License, which permits unrestricted use, distribution, and reproduction in any medium, provided the original work is properly cited.

Objectives. The study aimed at describing characteristics and outcome of tuberculous meningitis (TBM) in HIV-positive patients and comparing these parameters with those of extrapulmonary TB (TBEP) and pulmonary TB (TBP). Methods. Kaplan-Meier estimation and Poisson regression models were used to assess the mortality following TB diagnosis and to evaluate potential prognostic factors for the 3 groups of TB patients separately. Results. A total of 100 patients with TBM, 601 with TBEP, and 371 TBP were included. Patients with TBM had lower CD4 cell counts and only $17.0 \%$ received antiretroviral therapy (ART) at TB diagnosis. The cumulative probability of death at 12 months following TB was 51.2\% for TBM (95\% CI 41.4-61.6\%), $12.3 \%$ for TBP (8.9-15.7\%), and 19.4\% for TBEP (16.1-22.6) ( $P<0.0001$; log-rank test). For TBM, factors associated with a poorer prognosis were not being on ART (adjusted incidence rate ratio (aIRR) 4.00 (1.72-9.09), a prior AIDS diagnosis (aIRR = 4.82 (2.61-8.92)), and receiving care in Eastern Europe $($ IRR $=5.41(2.58-11.34))$ ). Conclusions. TBM among HIV-positive patients was associated with a high mortality rate, especially for patients from Eastern Europe and patients with advanced HIV-infection, which urgently calls for public health interventions to improve both TB and HIV aspects of patient management. 


\section{Introduction}

Tuberculous meningitis (TBM) is one of the most devastating manifestations of extrapulmonary tuberculosis (TBEP) and is associated with severe morbidity and high mortality [1]. HIV-positive individuals are known to be at increased risk of developing TBEP including TBM, particularly those patients with more advanced levels of immunodeficiency [24]. Further, it has been shown that TBM among HIV-positive patients in the period prior to combination antiretroviral therapy (cART) had higher mortality rates compared to HIVnegative individuals $[3,5]$.

Establishment of a TB diagnosis in HIV-positive patients poses a clinical challenge. This is true for both pulmonary TB (TBP) and TBEP and perhaps especially so for a TBM diagnosis [6]. Early diagnosis is important because it improves the overall prognosis; however, identification of acid-fast bacilli in the cerebrospinal fluid (CSF) and culture of Mycobacterium tuberculosis $(M t b)$ lacks sensitivity, and therefore a TBM diagnosis is often based on clinical suspicion combined with empirical decision making $[3,7]$.

No randomized controlled trials have investigated the optimal treatment regimen for TBM. Current treatment recommendations of 9-12 months of anti-TB treatment are extrapolated from clinical trials of treatment for TBP, with some guidelines advising a regimen consisting of rifampicin, isoniazid, pyrazinamide, and ethambutol [8], while others recommending that ethambutol can be replaced by either streptomycin or ethionamide $[9,10]$.

The overall aim of this study was to describe the patient characteristics, TB regimens, treatment outcomes, and prognostic factors for HIV-positive patients with TBM and compare these parameters to those of patients with TBEP or TBP.

\section{Methods}

2.1. The HIV/TB Study. The HIV/TB study is a retrospective, observational cohort study based on collaboration between fifty four clinics and cohorts in Eastern Europe (EE), Central/Northern Europe (CNE), Southern Europe (SE), and Argentina (AR). The study included patients of 16 years or older who were diagnosed with TB between January 2004 and December 2006 and who were either known to be HIVpositive or diagnosed with HIV up to 3 months after their TB diagnosis. Information was collected on standardized Case Report Forms (CRFs, available at http://www.cphiv.dk), and details of the study have previously been published [11]. In this paper, we describe characteristics and outcomes for patients with TBM, TBP, and TBEP.

Patients were categorized as having TBP if TB disease was limited to either the lungs, pleura, or both. TBEP included cases with either TB limited to at least one extrapulmonary organ system (and potentially involving lungs and pleura), including miliary TB or $M t b$ isolated from blood or bone marrow. Disseminated TB was defined as involvement of at least two extrapulmonary organ systems. Patients with TBM were categorized separately regardless of having additional pulmonary or other extrapulmonary involvement. The TBM diagnosis was established by evidence of mycobacteria in
CSF (culture/AFB/PCR positive in CSF) or by empirical decision of the clinician primarily based on a combination of central nervous system symptoms (e.g., headache, altered consciousness, dizziness, and nausea) and contemporaneous documentation of extracranial TB.

Patients were categorized as having either a definite TB diagnosis (culture/PCR positive), a probable TB diagnosis (positive microscopy for acid-fast-bacilli (AFB)/granulomatous inflammation on histology), or a presumptive TB diagnosis (based on clinical suspicion, where treatment was initiated and not subsequently stopped because the TB diagnosis was ruled out) as previously reported [11].

TB disease outcomes were classified as either "success," defined as either cure or treatment completed, or "fail," defined as either treatment failure, treatment deferred, treatment interrupted, death, or loss to follow-up [12].

2.2. Statistical Methods. Descriptive statistics were used to describe patients' characteristics in the three TB disease groups. Continuous variables were described using medians and interquartile ranges, categorical variables using numbers and percentages, and compared between diagnosis groups using Mann-Whitney and $\chi^{2}$-tests, respectively.

The date of TB diagnosis (baseline) was either the date when a positive TB sample was obtained or the date when TB treatment was initiated, whichever came first.

Kaplan-Meier estimation was used to describe the proportion of patients who had died following TB diagnosis, stratified by type of TB. Poisson regression models were used to evaluate potential prognostic factors for the three groups of TB patients separately, and the model included the following variables that were identified a priori: geographical region, age, CD4 cell count, usage of CART at time of TB, prior AIDS, certainty of the TB diagnosis, presence of disseminated TB disease, presence of multidrug resistant (MDR) TB, and usage of a rifamycin in the initial anti-TB regimen. As the focus was type of $\mathrm{TB}$, interactions between type of $\mathrm{TB}$ and other variables were investigated to determine if the relationships differed, for example, if the relationship between age and outcome differed depending on the type of TB diagnosis, and stratified results presented.

Sensitivity analyses were performed to investigate whether consistent results were found among patients with a definitive diagnosis compared to those with a presumptive or possible diagnosis and also to determine the relationship between type of TB diagnosis and death in the first 12 months of follow-up, where mortality is known to be the worst, particularly in Eastern Europe [13].

Follow-up was until date of death or the last date when the patient was known to be alive or July 2010, whichever occurred first. All analyses were performed using SAS, version 9.3 (Statistical analysis Software, version 9.3, Cary, NC, USA).

2.3. Ethical Approval. The overall study protocol was approved by local ethics committees for each of the participating clinics and cohorts as per local regulations and was registered with the Danish Data Protection Agency. 


\section{Results}

3.1. Patient Characteristics and Treatment. Of the 1072 patients with a classifiable TB diagnosis, 100 (9.3\%) were diagnosed with TBM, 371 (34.6\%) with TBP, and 601 (56.1\%) with TBEP. HIV and TB clinical characteristics of the patients are presented in Table 1. The proportion of TB patients presenting with TBM varied between Europe and Argentina (10.4\%, $10.4 \%$, and $8.3 \%$ in Eastern, Central/Northern, and Southern Europe, respectively compared with $4.3 \%$ in Argentina, $P=$ 0.072).

The majority of patients, 981 (91.5\%), in all three groups were known to be HIV-positive at the time of TB diagnosis. Patients with TBM had significantly lower CD4 cell counts compared to those with TBEP and TBP $(P<0.0001)$. Significantly more patients with TBM had a prior AIDS diagnosis other than TB: 39 (40.6\%) compared to $112(34.0 \%)$ TBP and 161 (29.0\%) TBEP patients $(P=0.043)$ (Table 1). A significantly higher proportion of patients with TBM had an AIDS defining illness within 3 months before or after their TB diagnosis, $33(33.0 \%)$ compared to 49 (13.2\%) patients with TBP and $89(14.8 \%)$ with TBEP $(P<0.0001)$, possibly reflecting their more advanced disease. In this time, only 3 AIDS defining illnesses occurred in $>10$ patients, oesophageal candidiasis $(n=84)$, pneumocystis jiroveci pneumonia $(n=25)$, and toxoplasmosis $(n=12)$, with other AIDS defining illnesses occurring in 50 patients. Patients with TBM were more likely to have one of the other, less frequently occurring AIDS defining illnesses (15/33, 45.5\%), compared to those with TBP $(13 / 49,26.5 \%)$ or TBEP $(22 / 89,24.7 \%, P=$ 0.078 ), although this was marginally statistically significant. Among the 15 other diagnoses in those with TBM, there were 4 diagnoses of AIDS dementia, 4 of HIV wasting, 3 of extraocular cytomegalovirus, and 1 each of cryptococcosis, cytomegalovirus retinitis, recurrent herpes simplex virus, and progressive multifocal leucoencephalopathy. Of those known to be HIV positive at time of TB diagnosis, 16 (16.7\%) of the TBM patients were receiving CART at time of TB diagnosis compared with $74(22.5 \%)$ and $87(15.7 \%)$ in the TBP and TBEP groups, $P=0.035$, and, of those with HIV-RNA measurements available at time of TB diagnosis, 7 (21.9\%) in the TBM group had a viral load $<400$ copies/mL, compared to $32(26.9 \%)$ of those with TBP and $27(13.8 \%)$ in the TBEP group $(P=0.015)$ (Table 1$)$.

The TB diagnosis was less often definite for TBM patients, 46 (46.0\%), compared with $244(65.8 \%)$ and $358(59.6 \%)$ in the TBP and TBEP groups, $P=0.0037$, and in $38(38.0 \%)$ patients $M t b$ was documented in cerebrospinal fluid (CSF) by microscopy (24), PCR (7), and/or culture (17). Severity of TB disease was similar in the TBM and TBEP groups: 77 (77\%) and $515(85.7 \%)$ had disseminated TB, respectively, and as per definition, no patients in the TBP group $(P=0.038$ between TBM and TBEP). Of the TBM and TBEP patients, $69(69.0 \%)$ and $478(79.5 \%)$ had TBP as well (Table 1$)$.

Significantly more patients with TBM were classified as having MDR-TB, 5 of $21(23.8 \%)$ TBM patients with DST available, compared to 14 of $96(14.7 \%)$ with TBP and 8 of 139 (5.8\%) with TBEP $(P=0.011)$. Significantly fewer patients with TBM were treated with an initial regimen containing

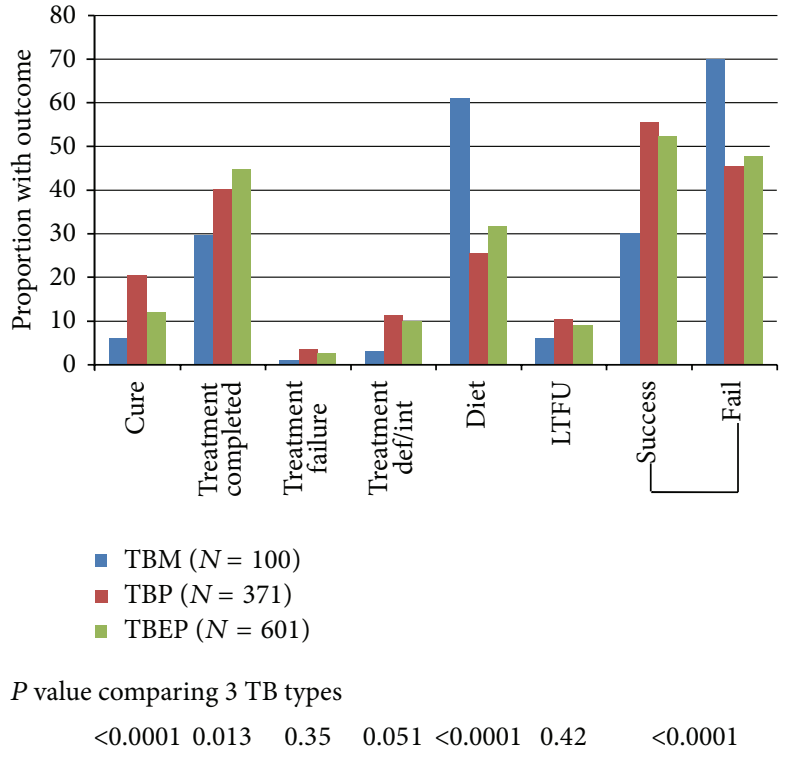

TBM: TB meningitis, TBP: pulmonary TB, and TBEP: extrapulmonary TB

FIGURE 1: Outcomes following TB diagnosis.

isoniazid, pyrazinamide, and rifamycin $(P=0.016)$ and more patients in the TBM group received at least one 2 nd line drug $(P=0.0007)$ as part of their initial regimen compared with the TBP and TBEP groups (Table 1).

3.2. Patient Outcome. Only $30(30.0 \%)$ patients with a TBM diagnosis attained successful treatment outcome (cure or treatment completed) compared to 204 (55.2\%) and 314 (52.3\%) in the TBP and TBEP groups, respectively, $P<$ 0.0001. Sixty-one patients in the TBM group died (61.0\%), 95 in the TBP group (25.6\%), and 191 in the TBEP group (31.8\%), $P<0.0001$ (Figure 1). The loss to follow-up rate during therapy was the lowest for TBM patients and overall below $10 \%$ (Figure 1). The overall mortality rate was considerably higher in the TBM group (39.3, 95\% CI 29.4-49.2 per 100 person years of follow-up (PYFU)) compared to the TBEP group (13.0, 95\% CI 11.2-14.7 per 100 PYFU) and the TBP group (6.4, 95\% CI 5.1-7.7 per 100 PYFU). 207 deaths (59.7\%) occurred within the first 12 months following TB diagnosis; this proportion was significantly higher for TBM (51, 83.6\%) compared to 44 (46.3\%) for TBP and 112 (58.6\%) for TBEP $(P<0.0001)$. Using Kaplan-Meier estimation, the cumulative probability of death at 12 months following TB diagnosis was $51.2 \%$ of those with TBM (95\% CI 41.4-61.6\%), compared to $12.3 \%$ for those with TBP (95\% CI 8.9-15.7\%) and $19.4 \%$ for those with TBEP (95\% CI 16.1-22.6) $(P<0.0001$, log-rank test) (Figure 2).

Table 2 shows the adjusted incidence rate ratios (aIRRs) for a priori selected variables within each of the TB groups. Patients from Eastern Europe (compared to all other regions combined) had an approximately 5-fold increased risk of dying following TBM, an 11-fold increased risk of dying following TBEP, and a 3-fold increased risk for dying following TBP (test for interaction between geographic region and TB 
TABLE 1: Baseline characteristics of HIV/TB coinfected patients according to the type of TB.

\begin{tabular}{|c|c|c|c|c|c|c|c|}
\hline & \multicolumn{2}{|c|}{ TB meningitis } & \multicolumn{2}{|c|}{ Pulmonary TB } & \multicolumn{2}{|c|}{ Extrapulmonary TB } & \multirow{2}{*}{ Chi-squared test } \\
\hline & $N$ & $\%$ & $N$ & $\%$ & $N$ & $\%$ & \\
\hline All & 100 & $(9.3)$ & 371 & $(34.6)$ & 601 & $(56.1)$ & \\
\hline \multicolumn{8}{|l|}{ HIV characteristics } \\
\hline \multicolumn{8}{|l|}{ Gender } \\
\hline Male & 74 & $(74.0)$ & 234 & $(63.1)$ & 422 & $(70.2)$ & \multirow{2}{*}{0.028} \\
\hline Female & 26 & $(26.0)$ & 137 & $(36.9)$ & 179 & $(29.8)$ & \\
\hline \multicolumn{8}{|l|}{ Race } \\
\hline White & 75 & $(75.0)$ & 224 & $(60.4)$ & 377 & $(62.7)$ & \multirow{2}{*}{0.026} \\
\hline Other & 25 & $(25.0)$ & 147 & $(39.6)$ & 224 & $(37.3)$ & \\
\hline \multicolumn{8}{|l|}{ Origin $^{1}$} \\
\hline Same country & 75 & $(78.1)$ & 300 & $(82.4)$ & 436 & $(74.8)$ & \multirow{3}{*}{0.0056} \\
\hline Other Europe & 6 & $(6.3)$ & 8 & $(2.2)$ & 13 & $(2.2)$ & \\
\hline Non-Europe & 15 & $(15.6)$ & 56 & $(15.4)$ & 134 & $(23.0)$ & \\
\hline \multicolumn{8}{|l|}{ HIV risk ${ }^{2}$} \\
\hline IDU & 55 & $(61.1)$ & 183 & $(52.4)$ & 279 & $(50.2)$ & \multirow{3}{*}{0.17} \\
\hline Heterosexual & 25 & $(27.8)$ & 118 & $(33.8)$ & 178 & $(32.0)$ & \\
\hline Other & 10 & $(11.1)$ & 48 & $(13.8)$ & 100 & $(17.9)$ & \\
\hline \multicolumn{8}{|l|}{ Region } \\
\hline Argentina & 5 & $(5.0)$ & 46 & $(12.4)$ & 64 & $(10.7)$ & \multirow{4}{*}{0.072} \\
\hline South & 17 & $(17.0)$ & 75 & $(20.2)$ & 114 & $(19.0)$ & \\
\hline Central & 17 & $(17.0)$ & 42 & $(11.3)$ & 105 & $(17.5)$ & \\
\hline East & 61 & $(61.0)$ & 208 & $(56.1)$ & 318 & $(52.9)$ & \\
\hline \multicolumn{8}{|l|}{$\mathrm{HBV}$} \\
\hline Negative & 33 & $(33.0)$ & 117 & $(31.5)$ & 243 & $(40.4)$ & \multirow{3}{*}{0.027} \\
\hline Positive & 5 & $(5.0)$ & 31 & $(8.4)$ & 51 & $(8.5)$ & \\
\hline Unknown & 62 & $(62.0)$ & 223 & $(60.1)$ & 307 & $(51.1)$ & \\
\hline \multicolumn{8}{|l|}{$\mathrm{HCV}$} \\
\hline Negative & 11 & $(11.0)$ & 58 & $(15.6)$ & 109 & $(18.1)$ & \multirow{3}{*}{0.044} \\
\hline Positive & 35 & $(35.0)$ & 106 & $(28.6)$ & 210 & $(34.9)$ & \\
\hline Unknown & 54 & $(54.0)$ & 207 & $(55.8)$ & 282 & $(46.9)$ & \\
\hline HIV before/at TB & & & & & & & \\
\hline Yes & 96 & $(96.0)$ & 329 & $(88.7)$ & 556 & $(92.5)$ & 0.027 \\
\hline HIV at least 3 months before $\mathrm{TB}^{3}$ & 72 & $(75.0)$ & 258 & $(78.4)$ & 396 & $(71.2)$ & 0.060 \\
\hline Prior AIDS $^{3}$ & & & & & & & \\
\hline Yes & 39 & $(40.6)$ & 112 & $(34.0)$ & 161 & $(29.0)$ & 0.043 \\
\hline On cART ${ }^{3}$ & 16 & $(16.7)$ & 74 & $(22.5)$ & 87 & $(15.7)$ & 0.035 \\
\hline Viral load $^{4}(<400 \mathrm{cp} / \mathrm{mL})$ & 7 & $(21.9)$ & 32 & $(26.9)$ & 27 & $(13.8)$ & 0.016 \\
\hline & Median & IQR & Median & IQR & Median & IQR & \\
\hline Age (years) & 33 & $28-40$ & 33 & $28-41$ & 33 & $28-39$ & 0.37 \\
\hline $\mathrm{HIV} \mathrm{VL}{ }^{4}\left(\log _{10} \mathrm{cp} / \mathrm{mL}\right)$ & 5.0 & $3.5-5.6$ & 4.5 & $2.6-5.3$ & 4.9 & $4.1-5.6$ & 0.0075 \\
\hline $\mathrm{CD} 4^{5}\left(/ \mathrm{mm}^{3}\right)$ & 113 & $40-269$ & 236 & $101-492$ & 143 & $58-304$ & $<0.0001$ \\
\hline Time HIV+ (months) & 42 & $3-87$ & 38 & $3-76$ & 35 & $30-70$ & 0.22 \\
\hline Date of TB diagnosis (month/year) & $12 / 05$ & $1 / 05-4 / 06$ & $6 / 05$ & $9 / 04-3 / 06$ & $8 / 05$ & $11 / 04-5 / 06$ & 0.017 \\
\hline TB characteristics & & & & & & & \\
\hline Prior $\mathrm{TB}^{6}$ & & & & & & & \\
\hline Yes & 6 & $(6.7)$ & 39 & $(11.5)$ & 44 & $(7.7)$ & 0.11 \\
\hline Disseminated TB & 77 & $(77.0)$ & - & - & 515 & $(85.7)$ & $0.038^{* *}$ \\
\hline Certainty of TB diagnosis & & & & & & & \\
\hline Definite & $46^{12}$ & $(46.0)$ & 244 & $(65.8)$ & 358 & $(59.6)$ & \\
\hline Probable & 24 & $(24.0)$ & 43 & $(11.6)$ & 96 & $(16.0)$ & 0.0037 \\
\hline Presumptive & 30 & $(30.0)$ & 84 & $(22.6)$ & 147 & $(24.5)$ & \\
\hline
\end{tabular}


TABLE 1: Continued.

\begin{tabular}{|c|c|c|c|c|c|c|c|}
\hline & \multicolumn{2}{|c|}{ TB meningitis } & \multicolumn{2}{|c|}{ Pulmonary TB } & \multicolumn{2}{|c|}{ Extrapulmonary TB } & \multirow{2}{*}{ Chi-squared test } \\
\hline & $N$ & $\%$ & $N$ & $\%$ & $N$ & $\%$ & \\
\hline \multicolumn{8}{|l|}{ Symptoms } \\
\hline Yes & 99 & $(99.0)$ & 345 & $(93.0)$ & 577 & $(96.0)$ & 0.018 \\
\hline Fever & 81 & $(81.8)$ & 269 & $(78.6)$ & 490 & $(84.8)$ & 0.033 \\
\hline Weight loss & 51 & $(51.5)$ & 177 & $(51.3)$ & 342 & $(59.3)$ & 0.041 \\
\hline Cough with expectorate & 26 & $(26.3)$ & 229 & $(66.4)$ & 201 & $(34.8)$ & $<0.0001$ \\
\hline Dry cough & 12 & $(12.1)$ & 59 & $(17.1)$ & 132 & $(22.9)$ & 0.013 \\
\hline Other & 79 & $(79.8)$ & 161 & $(46.7)$ & 357 & $(61.9)$ & $<0.0001$ \\
\hline \multicolumn{8}{|l|}{ Anti-TB drug resistance } \\
\hline \multicolumn{8}{|l|}{ H-resistant ${ }^{7}$} \\
\hline Yes & 7 & $(33.3)$ & 24 & $(25.0)$ & 18 & $(13.0)$ & 0.016 \\
\hline \multicolumn{8}{|l|}{ Multidrug resistant $^{7}$} \\
\hline Yes & 5 & $(23.8)$ & 14 & $(14.7)$ & 8 & (5.8) & 0.011 \\
\hline \multicolumn{8}{|l|}{ Initial anti-TB treatment } \\
\hline RHZ & 51 & $(51.0)$ & 246 & $(66.3)$ & 367 & $(61.1)$ & 0.016 \\
\hline On 1st line drugs only ${ }^{8}$ & 35 & $(35.0)$ & 209 & $(56.3)$ & 307 & $(51.1)$ & 0.0007 \\
\hline \multirow[t]{2}{*}{ On 2nd line drug $(\mathrm{s})^{9}$} & 65 & $(65.0)$ & 162 & $(43.7)$ & 294 & $(48.9)$ & \\
\hline & Median & IQR & Median & IQR & Median & IQR & \\
\hline Time since prior $\mathrm{TB}^{10}$ (months) & 123 & $72-153$ & 46 & $32-71$ & 93 & $30-141$ & 0.017 \\
\hline Weight $^{11}(\mathrm{~kg})$ & 62 & $52-68$ & 61 & $53-69$ & 60 & $52-72$ & 0.95 \\
\hline
\end{tabular}

${ }^{1}$ Data available for 1043 (97.3\%) at baseline. ${ }^{2} 996$ (92.9\%) at baseline. ${ }^{3}$ Of 981 HIV positive at baseline. ${ }^{4} 347$ patients (35.4\%) of those HIV positive at baseline. ${ }^{5} 788(80.3 \%)$ of those HIV+ at baseline. ${ }^{6}$ Of those where information about prior TB diagnosis is known, $N=1001 .{ }^{7}$ Data on resistance available for 256 patients. ${ }^{8}$ Ethambutol or streptomycin plus RHZ. ${ }^{9}$ Any other TB drug. ${ }^{10}$ Of those with a previous TB diagnosis. ${ }^{11}$ Of $703(65.6 \%)$ with weight measured within 1 year of TB diagnosis. ${ }^{12} \mathrm{Mtb}$ documented in cerebrospinal fluid (CSF) for 38 patients-microscopy (24), PCR (7), and/or culture (17). H: isoniazid, R: rifamycin, and Z: pyrazinamide.

Baseline was defined as the date of TB diagnosis.

${ }^{*}$ Global $P$ values comparing all three disease groups (TBM, TBP, and TBEP).

${ }^{* *}$ The $P$ value compares TBM and TBEP (by definition TBP cannot be disseminated).

IDU: injection drug user, HBV: hepatitis B, HCV: hepatitis C, HIV before/at TB: HIV diagnosed before TB diagnosis, HIV at least 3 months before TB: HIV diagnosed at least 3 months before TB diagnosis, VL: viral load, and time HIV+: number of months the patients have been HIV+ (Median).

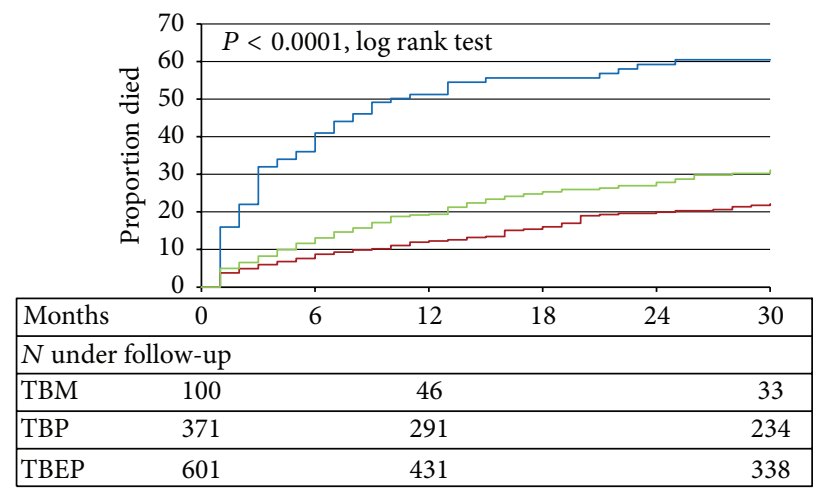

TBM: TB meningitis, TBP: pulmonary TB, and TBEP: extrapulmonary TB

$$
\begin{aligned}
& \text { TBM } \\
& - \text { TBP } \\
& - \text { TBEP }
\end{aligned}
$$

FIGURE 2: Kaplan-Meier progression to death.

group, $P=0.0004)$. Prior AIDS was strongly associated with death among TBM patients, and less pronounced so among TBEP patients, but not among TBP patients. Being on
cART at TB diagnosis was associated with approximately $2 / 3$ reduction in risk of dying in all three groups, whereas low CD4 cell count at time of TB was an independent prognostic factor for patients with TBP and TBEP but not for TBM patients. TB resistance and usage of a rifamycin containing initial regimen were not associated with death in any of the groups, perhaps at least partially due to low numbers in some of the groups.

3.3. Sensitivity Analyses. In a sensitivity analysis confined to patients with a definitive diagnosis, consistent results were found for all diagnoses to those shown in Table 2, although the confidence intervals became very wide because of the smaller number of patients included which makes any firm conclusions about consistency difficult. For example, there were 46 TBM patients with a definitive diagnosis of TB. Patients from Eastern Europe had almost a 3-fold increased incidence of death following TBM compared to patients from all other regions compared (aIRR 2.96; 95\% CI 1.09-8.06, $P=0.029$ ). Being on cART at TBM was associated with a lower risk of death (aIRR 0.25 ; 95\% CI 0.07-0.87, $P=0.017$ ). An analysis further limited to the 38 patients where $M t b$ was documented from the CSF showed similar results, but with even less precision (data not shown). 


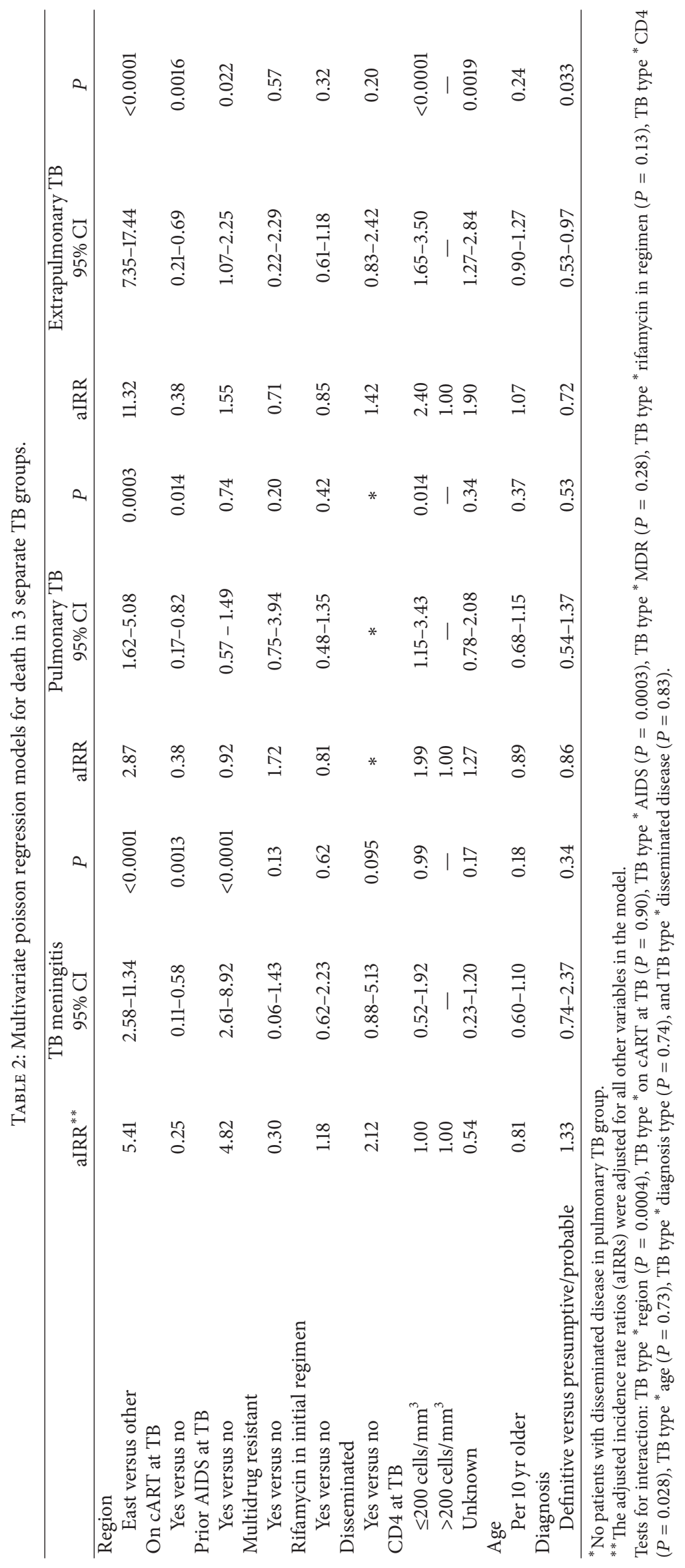


A sensitivity analysis was also performed limited to the first 12 months of follow-up. As expected, as the majority of patients with TBM died within 12 months, these results were highly consistent with those shown in Table 2 . The results were also highly consistent for TBP. In the first 12 months after diagnosis, there was less of a difference between regions for TBEP; after adjustment, patients from Eastern Europe had almost a 7-fold increased incidence of death compared to other regions (aIRR 6.74; 95\% CI 4.38-10.36, $P<0.0001$ ). The other results were consistent with those shown in Table 2.

\section{Discussion}

The present study demonstrates that among HIV-positive patients in Europe and Argentina, TBM is associated with a high mortality rate, which is significantly higher than among patients with either TBP or TBEP without meningeal involvement. Approximately 51\% of patients with TBM died within 12 months of TB diagnosis, and our overall findings are consistent with those from other continents [10]. A randomized, controlled trial from Vietnam found that among 96 TBM patients coinfected with HIV, approximately 65\% died within 9 months after anti-TB treatment had been initiated [3]. A retrospective study from Argentina found a mortality rate of $63 \%$ during hospitalization among 101 TBM patients coinfected with HIV [6], while a study from Zimbabwe documented 67\% in-hospital mortality among 21 culture positive TBM patients coinfected with HIV [14].

Patients included in the previous studies were characterized by severe immune suppression at the time of TBM diagnosis and none had received cART. In the present study, only $17 \%$ of TBM patients were on CART at TBM diagnosis, and the majority of patients with TBM were immunosuppressed. Factors which were significantly associated with death following TBM included region of follow-up (Eastern Europe), having a prior (non-TB) AIDS diagnosis, and not receiving cART.

Only $30 \%$ of TBM patients were classified as having treatment success defined as either cure or treatment completed. These numbers are worrisome and can primarily be explained by the very high mortality rate observed in this group. World Health Organization has set targets of reducing TB mortality rates by $50 \%$ by 2015 relative to 1990, a goal that is unlikely to be met in Eastern Europe-a region where both the TB and HIV epidemics are still increasing [15-18].

We have previously reported marked regional differences in outcome following a TB diagnosis in general among HIVpositive patients in Europe and Argentina. Approximately $30 \%$ of the patients in Eastern Europe died within the first year after a TB diagnosis, which is 4-5-fold higher than in Western Europe, but also 3-fold higher than in other middle-income countries such as Argentina [11]. The present study adds to the original report by documenting that these regional differences were also present for the three TB entities separately - and not least so for those presenting with extrapulmonary involvement, namely, TBM and TBEP, which in our setting to a large extent both could be characterized as disseminated TB disease.
Fifty-four clinics and cohorts in the European Region and Argentina contributed with data to this large study which has enabled identification of 100 clinical cases of TBM coinfected with HIV and included data from Eastern Europe, a region from where data remain scarce. Although TBM is a relatively rare disease, the size of the study has allowed for comparisons of characteristics and patient outcomes as well as analyzing regional differences in outcome.

Our study has several limitations. First of all, this was a retrospective study with observational data. Secondly, as an intrinsic limitation to TBM studies, TBM is a difficult diagnosis to establish and, consistent with other studies, only $38.0 \%$ of TBM cases were based on positive findings in the CSF. Further, the present study did not collect data on CSF-leucocyte count, CSF protein, and CSF glucose, which would have provided data to support the TBM diagnosis in patients with negative cultures. Additionally, as relevant differential diagnoses, 33\% of TBM patients were diagnosed with other opportunistic infections around time of the TB diagnosis, including brain toxoplasmosis, progressive multifocal leucoencephalopathy and AIDS dementia. Finally, we cannot rule out that the loss of follow-up rate could have impacted the results, as also discussed previously [11]. Sensitivity analyses limited to the patients with a definite TB diagnosis and including only the first 12 months of follow-up showed consistent results with the main findings.

A large prospective observational cohort study of HIVpositive patients presenting with $\mathrm{TB}$ is currently recruiting patients in Europe and Latin America to help our understanding of how to improve the outcome for HIV- and TBcoinfected patients including those with most severe disease such as TBM [19].

\section{Conclusion}

In summary, HIV-positive patients with TBM in Europe and Argentina are characterized by severe immune suppression and by an alarmingly high mortality rate. The poor prognosis following TBM is especially true for patients from Eastern Europe and for patients with advanced HIV-infection, which urgently calls for public health interventions to improve patient management in terms of earlier diagnosis of HIVinfection, timely and accurate TB diagnosis, optimal TB treatment, and access to cART.

\section{Conflict of Interests}

The authors declare that there is no conflict of interests regarding the publication of this paper.

\section{Acknowledgments}

The role of each of the members of the writing group: Anne Marie W. Efsen contributed with data analyses and interpretation, and was responsible for writing the paper. Daniel Grint and Amanda Mocroft performed data analyses and contributed with ideas for data analyses and writing the paper. Daria N. Podlekareva contributed with project development 
and coordination, data analyses and interpretation, and writing the paper. Frank A. Post, Robert F. Miller, and Jose M. Miro contributed with national coordination, data collection, ideas for data analyses, and writing the paper, and Anna Vassilenko, Hansjakob Furrer, Mathias Bruyand, Alexander M. Panteleev, Aza Rakhmanova, Enrico Girardi, Marcelo H. Losso, Indra Zeltina, Joan Caylá, Jens D. Lundgren, and Niels Obel contributed with national coordination, data collection, and with writing the paper. Ole Kirk contributed with ideas for the study design, overall coordination, and data analysis and interpretation, with writing paper and supervised the study. Data collection in Eastern Europe (Belarus, Latvia, Russia, and Ukraine) and Argentina was funded by the Copenhagen HIV Programme and the EuroSIDA study. Primary support for EuroSIDA is provided by the European Commission BIOMED 1 (CT94-1637), BIOMED 2 (CT972713), the 5th Framework (QLK2-2000-00773), and the 6th Framework (LSHP-CT-2006-018632) programs. Current support also includes unrestricted Grants from Bristol-Myers Squibb, GlaxoSmithKline, Roche, Gilead, Pfizer, Merck and Co., Tibotec, and Boehringer-Ingelheim. The participation of centers from Switzerland was supported by a Grant from the Swiss Federal Office for Education and Science. Data collection in Western Europe was self-funded by the participating cohorts as follows: Aquitaine Cohort, France; Danish HIV Cohort, Denmark; SWISS HIV Cohort, Switzerland; and Mortimer Market Hospital and King's College Hospital in London, UK. In Spain, the study was funded by Ministerio de Sanidad y Consumo, Instituto de Salud Carlos III, Spanish Network for the AIDS Research (RD06/006), Madrid, Spain, and Agencia de Salud Pública de Barcelona. In Rome, INMI Spallanzani, data collection was supported in part by a Grant from the Italian Ministry of Health (Progetto AIDS, no. $40 \mathrm{H} 79$ ) to EG. The study group (participating centers with their country names) consists of the following: Argentina, Buenos Aires: Marcelo H. Losso (principal investigator); J. J. Toibaro (Project Manager); Hospital Interzonal General de Agudos DR. D. PAROISSIEN: E. Warley, N. Tamayo, and M. Cristina Ortiz; Hospital General de Agudos Donación F. Santojanni: P. Scapelatto and E. Bottaro; Hospital Provincial Petrona V. de Cordero San Fernando: F. Murano; Hospital San Juan de Dios (La Plata): M. Miachans, J. Contarelli, and L. Massera; Hospital Interzonal HIGA Oscar Alende (Mar del Plata): J. Corral, M. Hualde, and C. Miglioranza; Hospital de Infecciosas Francisco Muñiz: M. Corti and H. Metta; Hospital General de Agudos Dr T. Álvarez: A. Casiró and R. Cuini; Hospital Posadas: H. Laplume; Hospital Rawson (Cordoba): D. David and C. Marson; C.A.I.C.I: S. Lupo and L. Trape; Hospital Piñero: O. Garcia Messina and O. Gear; Hospital General de Agudos J. M. Ramos Mejía: J. J. Toibaro and J. M. Bruguera. Belarus, Minsk: University Hospital of Infectious Diseases: I. Karpov (principal investigator) and A. Vassilenko; Belorussian State Medical University: E. Skrahina, A. Skrahin; Gomel: University Hospital of Infectious Diseases: S. Zhavoronok, V. Mitsura, and D. Ruzanov; University Hospital of Tuberculosis: V. Bondarenko; Svetlogorsk: Gomel Region AIDS centre: O. Suetnov and D. Paduto; Denmark: Danish HIV Cohort: N. Obel (principal investigator); Rigshospitalet: J. Gerstoft; Hvidovre University Hospital: G. Kronborg;
Odense University Hospital: C. Pedersen; Aarhus University Hospitals, Skejby: C. S. Larsen; Aalborg Hospital: G. Pedersen; Herning Hospital: A. L. Laursen; Helsingør Hospital: L. Nielsen; Kolding Hospital: J. Jensen; France: Aquitaine Cohort: F. Dabis (principal investigator); for Epidemiology: G. Chêne, F. Dabis, S. Lawson-Ayayi, M. Bruyand, R. Thiébaut and L. Wittkop. Bordeaux hospital: Professor P. Morlat (Professor F. Bonnet, Drs N. Bernard, M. Hessamfar, D. Lacoste and MA. Vandenhende); Professor M. Dupon (Drs FA. Dauchy and H. Dutronc), Professor M. Longy-Boursier (Professor P. Mercié and Drs P. Duffau and J. Roger Schmeltz), Professor D. Malvy (Drs T. Pistone and MC Receveur), Professor D. Neau (Drs C. Cazanave, A. Ochoa, and MO. Vareil), Professor JL. Pellegrin (Professor JF. Viallard, C. Greib, and E. Lazaro); Professor H. Fleury (Professor ME. Lafon, Drs S. Reigadas and P. Trimoulet); Professor D. Breilh; Professor M. Molimard (Drs S. Bouchet and K. Titier); Professor JF. Moreau (Dr I. Pellegrin); Drs F. Haramburu, G. Arcachon hospital: A. Dupont, Dax Hospital: Y. Gerard (L. Caunègre and K. André); Bayonne Hospital: F. Bonnal (S. Farbos, M. C. Gemain); Libourne Hospital: J. Ceccaldi (S. Tchamgoué); Mont de Marsan Hospital: S. De Witte (K. Courtault); Pau hospital: E. Monlun (V. Gaborieau); Périgeux hospital: P. Lataste (JP. Meraud); Villeneuve-sur-Lot hospital: I. Chossat. Italy from Brescia: Institute of Infectious and Tropical Diseases: A. C. Carvalho, R. Basché; I Division of Infectious Diseases, Spedali Civili: I. E. Hamad, B. A. Ricci, Bergamo: F. Maggiolo, V. Ravasio; Modena: Clinica di Malattie Infettive, C. Mussini (principal investigator), F. Prati, S. Castelletti; Rome, INMI L. Spallanzani: A. Ammassari, A. Antinori, R. Bellagamba, E. Busi Rizzi, S. Cicalini, A. Corpolongo, A. Capaldo, A. Di Caro, E. Girardi (principal investigator), D. Goletti, S. Grisetti, G. Gualano, F. N. Lauria, L. Parracino, F. Palmieri, N. Petrosillo, C. Pinetti, and A. Sampaolesi; ICONA cohort: Board of Directors: M. Moroni (Chair), G. Angarano, A. Antinori, O. Armignacco, A. d'Arminio Monforte, F. Castelli, R. Cauda, G. Di Perri, M. Galli, R. Iardino, G. Ippolito, A. Lazzarin, C.F. Perno, F. von Schloesser, and P. Viale; Scientific Secretariat: d'Arminio Monforte, A. Antinori, A. Castagna, F. Ceccherini-Silberstein, A. Cozzi-Lepri, E. Girardi (principal investigator), S. Lo Caputo, C. Mussini, and M. Puoti; Steering Committee: M. Andreoni, A. Ammassari, A. Antinori, A. d’Arminio Monforte, C. Balotta, P. Bonfanti, S. Bonora, M. Borderi, MR. Capobianchi, A. Castagna, F. Ceccherini-Silberstein, A. Cingolani, P. Cinque, A. CozziLepri, A. De Luca, A. Di Biagio, E. Girardi, N. Gianotti, A. Gori, G. Guaraldi, G. Lapadula, M. Lichtner, S. Lo Caputo, G. Madeddu, F. Maggiolo, G. Marchetti, S. Marcotullio, L. Monno, C. Mussini, M. Puoti, E. Quiros Roldan, and S. Rusconi; Statistical and Monitoring Team: A. Cozzi-Lepri, P. Cicconi, I. Fanti, T. Formenti, L. Galli, and P. Lorenzini; Participating Physicians and Centers: A. Giacometti and A. Costantini (Ancona); G. Angarano, L. Monno, and C. Carrisa (Bari); F. Maggiolo and C. Suardi (Bergamo); P. Viale, E. Vanino, and G. Verucchi (Bologna); F. Castelli, E. Quiros Roldan, and C. Minardi (Brescia); T. Quirino and C. Abeli (Busto Arsizio); P. E. Manconi and P. Piano (Cagliari); J. Vecchiet, K. Falasca (Chieti); L. Sighinolfi, D. Segala (Ferrara); F. Mazzotta, S. Lo Caputo (Firenze); G. Cassola, G. 
Viscoli, A. Alessandrini, R. Piscopo, G. Mazzarello (Genova); C. Mastroianni, V. Belvisi (Latina); P. Bonfanti, I. Caramma (Lecco); A. P. Castelli (Macerata); M. Galli, A. Lazzarin, G. Rizzardini, M. Puoti, A. d'Arminio Monforte, A. L. Ridolfo, R. Piolini, A. Castagna, S. Salpietro, L. Carenzi, M. C. Moioli, P. Cicconi, and G. Marchetti (Milano); C. Mussini and C. Puzzolante (Modena); A. Gori and G. Lapadula (Monza); N. Abrescia, A. Chirianni, M.G. Guida, and M. Gargiulo (Napoli); F. Baldelli and D. Francisci (Perugia); G. Parruti, T. Ursini (Pescara); G. Magnani, M. A. Ursitti (Reggio Emilia); R. Cauda, M. Andreoni, A. Antinori, V. Vullo, A. Cingolani, A. d'Avino, A. Ammassari, L. Gallo, E. Nicastri, R. Acinapura, M. Capozzi, R. Libertone, and G. Tebano (Roma); A. Cattelan (Rovigo); M. S. Mura, G. Madeddu (Sassari); P. Caramello, G. Di Perri, G. C. Orofino, S. Bonora, M. Sciandra (Torino); G. Pellizzer, V. Manfrin (Vicenza). Latvia, Riga: State Agency of TB and Lung Diseases: V. Riekstina (principal investigator); Infectology Centre of Latvia: P. Aldins. Romania, Bucharest: Spitalul de Boli Infectioase si Tropicale: D. Duiculescu (principal investigator). Russia, St. Petersburg: Botkin Hospital of Infectious Diseases: A. Rakhmanova (principal investigator), E. Malashenkov, A. Kozlov, St. Petersburg City TB Hospital No. 2, A. Panteleev; Novgorod City AIDS Centre: S. Buzunova. Spain, Barcelona: Hospital Clinic, IDIBAPS, University of Barcelona J. M. Miro (principal investigator), J. F. García-Goez, A. MorenoCamacho, J. A. Martínez, J. González, F. García-Alcaide, E. de Lazzari, J. M. Gatell; Hospital del Mar: P. Sanchez, J. L. LopezColomes. Mutua de Terrassa: X. Martínez-Lacasa. Hospital Universitari Vall d'Hebrón: V. Falcó, A. Imaz, I. Ocaña, R. Vidal. Hospital Universitari de Sant Pau: M. A. Sambeat. Agencia de Salud Pública de Barcelona: J. Caylà, A. MorenoMartínez, A. Orcau. Switzerland, Swiss HIV Cohort: Zurich: R. Weber (principal investigator); Basel: M. Battegay; Geneva: B. Hirschel; Lausanne: M. Cavassini; Lugano: E. Bernasconi; St Gall: P. Schmid; Bern: H. Furrer; Data Center Lausanne: M. Rickenbach; United Kingdom, London: King's Hospital: F. Post, L. Campbell; Mortimer Market Centre: R. Miller and A. Arenas-Pinto; Ukraine: Kiev City AIDS Centre: N. Chentsova (principal investigator); EuroSIDA: J. D. Lundgren; Coordinating Centre (CHIP): A. W. Efsen, D. Podlekareva, O. Kirk, D. Grint, A. Mocroft, J. Kjær. Steering Committee: N. Chentsova, H. Furrer, E. Girardi, M. Bruyand, M. H. Losso, J. D. Lundgren, A. Panteleev, R. Miller, J. M. Miro, N. Obel, F. Post, V. Riekstina, A. Skrahin, and J. J. Toibaro.

\section{References}

[1] G. E. Thwaites, N. D. Bang, N. H. Dung et al., "Dexamethasone for the treatment of tuberculous meningitis in adolescents and adults," The New England Journal of Medicine, vol. 351, no. 17, pp. 1741-1751, 2004.

[2] J. Berenguer, S. Moreno, F. Laguna et al., "Tuberculous meningitis in patients infected with the human immunodeficiency virus," The New England Journal of Medicine, vol. 326, no. 10, pp. 668-672, 1992.

[3] G. E. Thwaites, N. D. Bang, N. H. Dung et al., "The influence of HIV infection on clinical presentation, response to treatment and outcome in adults with tuberculous meningitis," Journal of Infectious Diseases, vol. 192, no. 12, pp. 2134-2141, 2005.
[4] C. Vinnard and R. R. MacGregor, "Tuberculous meningitis in HIV-infected individuals," Current HIV/AIDS Reports, vol. 6, no. 3, pp. 139-145, 2009.

[5] S. M. Katrak, P. K. Shembalkar, S. R. Bijwe, and L. D. Bhandarkar, "The clinical, radiological and pathological profile of tuberculous meningitis in patients with and without human immunodeficiency virus infection," Journal of the Neurological Sciences, vol. 181, no. 1-2, pp. 118-126, 2000.

[6] D. Cecchini, J. Ambrosioni, C. Brezzo et al., "Tuberculous meningitis in HIV-infected and non-infected patients: comparison of cerebrospinal fluid findings," International Journal of Tuberculosis and Lung Disease, vol. 13, no. 2, pp. 269-271, 2009.

[7] G. E. Thwaites, T. T. H. Chau, K. Stepniewska et al., "Diagnosis of adult tuberculous meningitis by use of clinical and laboratory features," The Lancet, vol. 360, no. 9342, pp. 1287-1292, 2002.

[8] American Thoracic Society CfDC, and infectious Diseases Society of America, "Treatment of Tuberculosis," MMWR Recommendations and Reports RR-11, 2003, http://www.cdc.gov/ mmwr/PDF/rr/rr5211.pdf.

[9] P. Ormerod, I. Campbell, V. Novelli et al., "Chemotherapy and management of tuberculosis in the United Kingdom: recommendations 1998," Thorax, vol. 53, no. 7, pp. 536-548, 1998.

[10] S. Marais, D. J. Pepper, B. J. Marais, and M. E. Török, "HIVassociated tuberculous meningitis: diagnostic and therapeutic challenges," Tuberculosis, vol. 90, no. 6, pp. 367-374, 2010.

[11] D. N. Podlekareva, A. Mocroft, F. A. Post et al., "Mortality from HIV and TB coinfections is higher in Eastern Europe than in Western Europe and Argentina," Acquired Immune Deficiency Syndrome, vol. 23, no. 18, pp. 2485-2495, 2009.

[12] J. Veen, M. Raviglione, H. L. Rieder et al., "Standardized tuberculosis treatment outcome monitoring in Europe," European Respiratory Journal, vol. 12, no. 2, pp. 505-510, 1998.

[13] D. N. Podlekareva, A. M. Panteleev, D. Grint et al., "Short and long term mortality and causes of death in HIV/TB patients in Europe," European Respiratory Journal. In press.

[14] J. G. Hakim, I. T. Gangaidzo, R. S. Heyderman et al., "Impact of HIV infection on meningitis in Harare, Zimbabwe: a prospective study of 406 predominantly adult patients," Acquired Immune Deficiency Syndrome, vol. 14, no. 10, pp. 1401-1407, 2000.

[15] F. F. Hamers and A. M. Downs, "HIV in central and eastern Europe," The Lancet, vol. 361, no. 9362, pp. 1035-1044, 2003.

[16] S. D. Lawn and A. I. Zumla, “Tuberculosis," The Lancet, vol. 378, no. 9785, pp. 57-72, 2011.

[17] WHO, “Global tuberculosis report," Tech. Rep., 2012, http:// apps.who.int/iris/bitstream/10665/75938/1/9789241564502_eng .pdf.

[18] WHO TSTS, Building on and enhancing DOTS to meet the TBrelated Millenium Development Goals, 2006.

[19] “TB:HIV”, study as part of the EuroCoord study, 2013, http:// www.cphiv.dk/TBHIV/tabid/284/Default.aspx. 


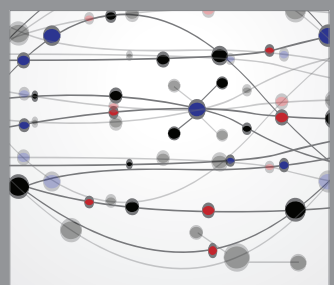

The Scientific World Journal
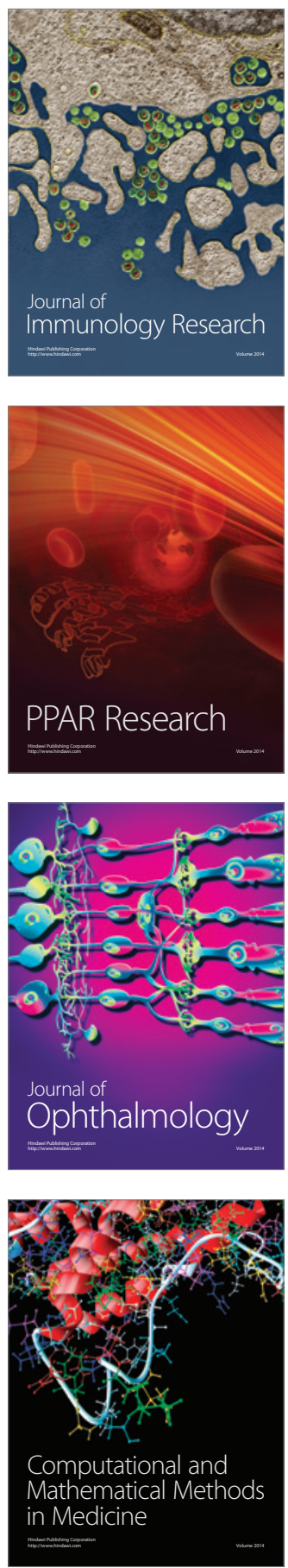

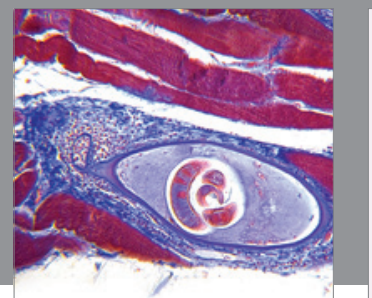

Gastroenterology

Research and Practice
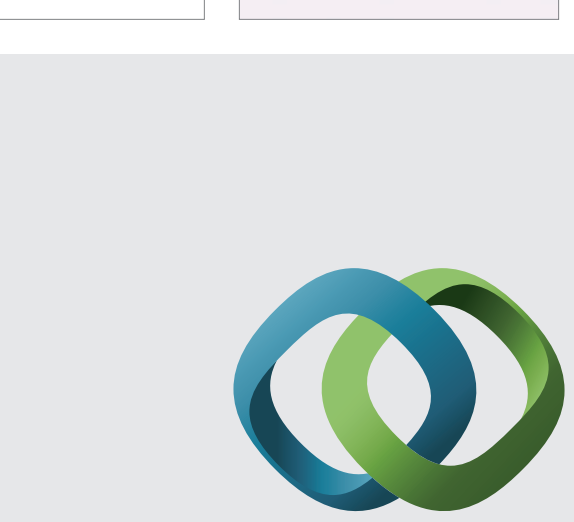

\section{Hindawi}

Submit your manuscripts at

http://www.hindawi.com
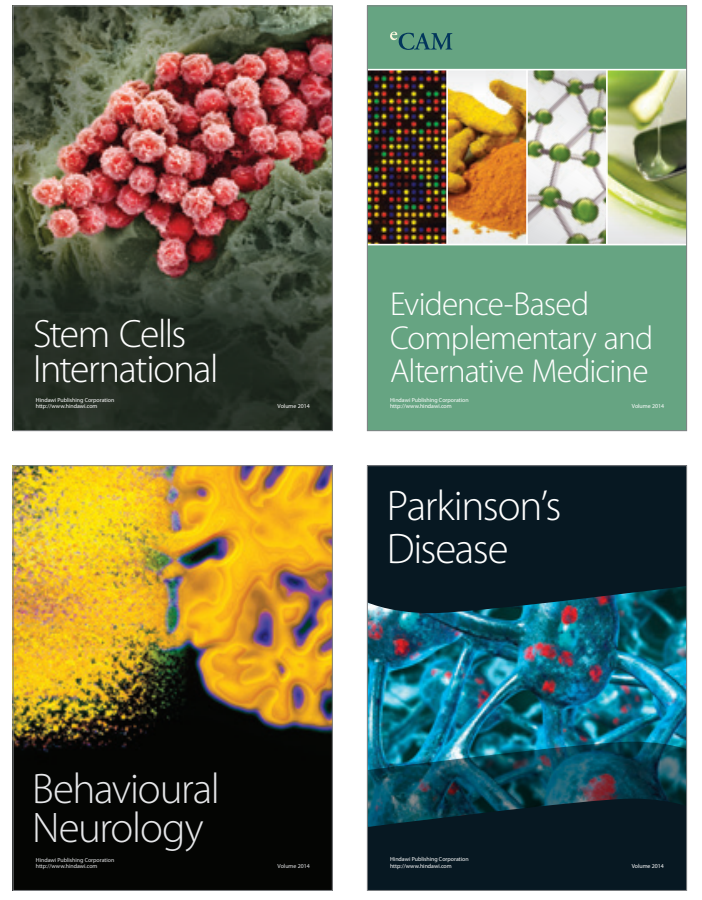
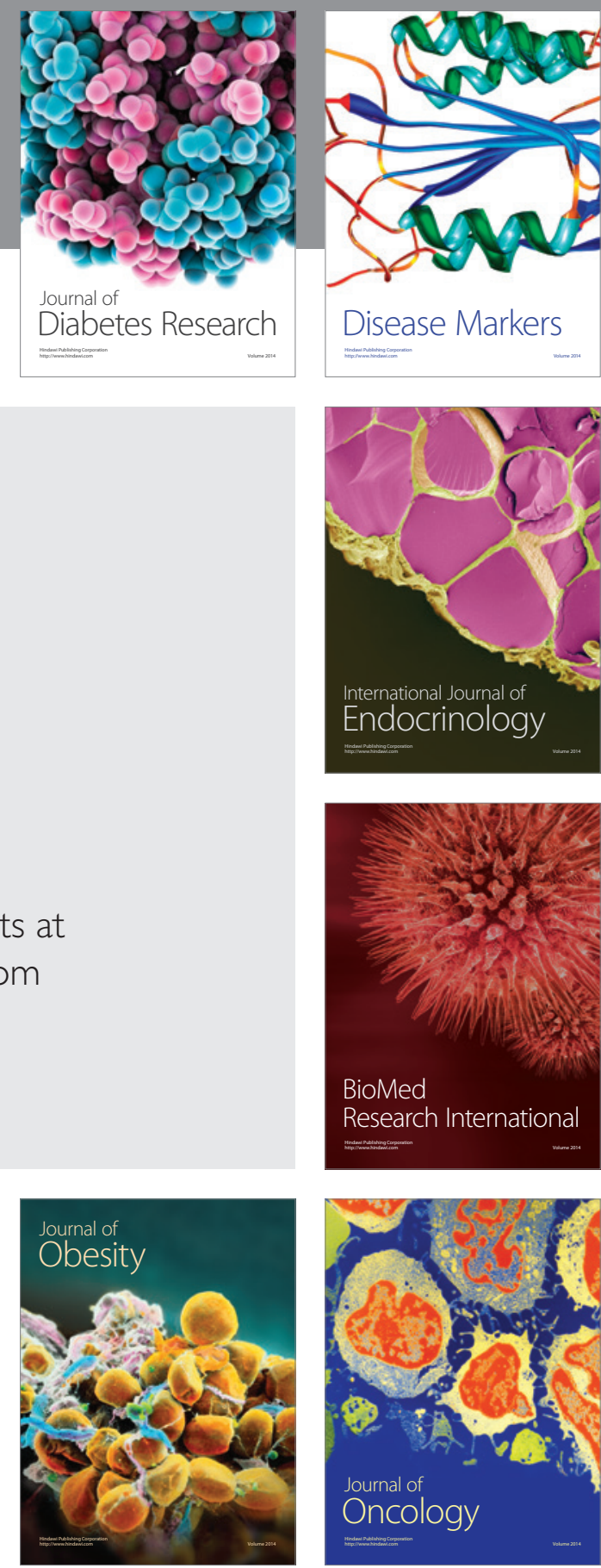

Disease Markers
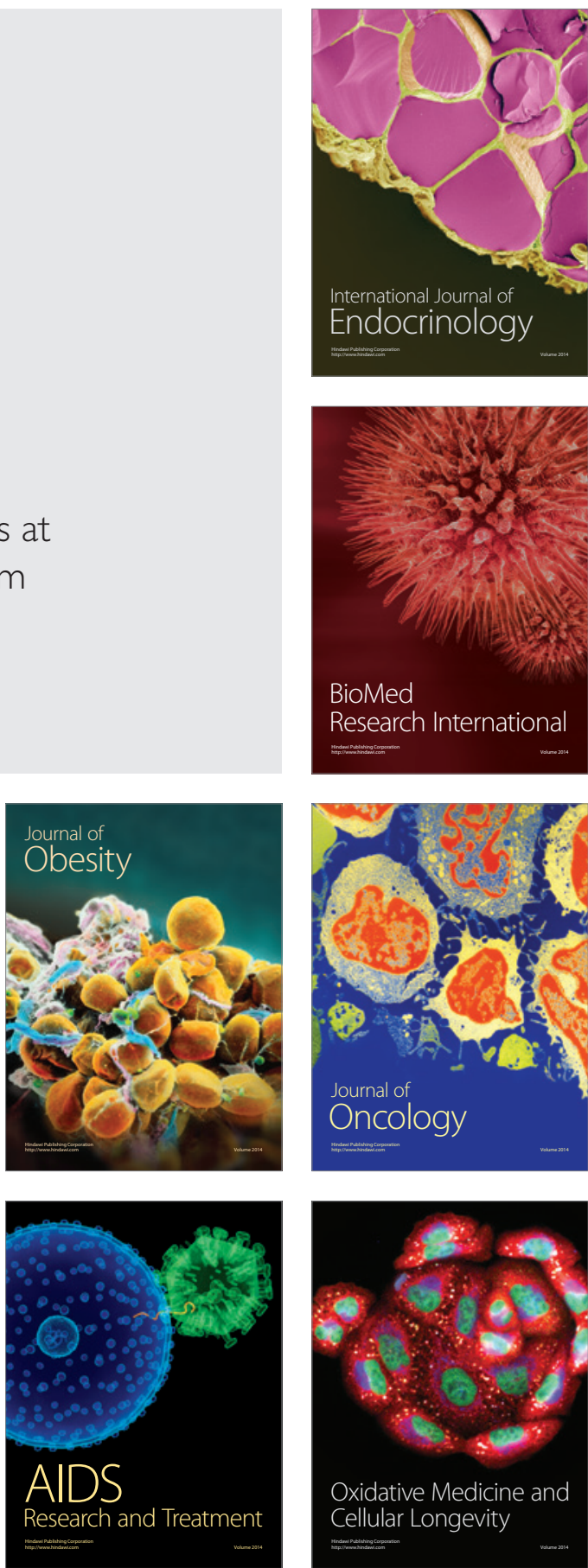